Fecha de recepción: diciembre 2018 Fecha de aceptación: febrero 2019 Versión final: mayo 2019

\section{Colores Sustentables: Cuando Ciencia y Diseño se Encuentran}

Carlos Fiorentino *

Resumen: Este artículo se basa en el trabajo realizado como proyecto de doctorado del autor, y se ha nutrido de la idea del biocentrismo para generar nuevas espacios de encuentro entre el diseño y las ciencias que estudian la naturaleza, en particular sobre maneras sustentables de producir color. Como parte de este proyecto se han creado herramientas de integración multidisciplinaria que podrían contribuir a desarrollar mejores métodos de investigación y trabajo para la biomimesis, así como también nuevos canales de comunicación entre científicos y diseñadores con interés en implementar la biomimesis de manera tangible. Este artículo describe el proceso que conllevó este proyecto y ofrece reflexiones sobre presente y futuro de esta experiencia.

Palabras clave: Color estructural - diseño biocéntrico - antropocentrismo - biomimesis sustentabilidad - multidisciplinaridad - prototipeado académico - rich prospect browsing.

[Resúmenes en inglés y portugués en las páginas 86 - 87]

${ }^{(*)}$ BDes, MDes, PhD Candidate in Human Ecology. Universidad de Alberta, Canada

\title{
Introducción
}

El encuentro entre disciplinas muy diferentes en sus metodologías, en sus epistemologías y en sus habituales áreas de influencia, suele dar pocos resultados tangibles, más allá de eventuales sinergias más cortoplacistas que prospectivas, innovadoras, y generadoras de nuevas teorías. En particular las ciencias naturales como la biología y la física no comparten con las disciplinas del diseño una misma manera de entender y aplicar el conocimiento, ni tampoco las disciplinas de diseño pueden enfocarse en la rigurosidad de la investigación científica; ambas "maneras de saber" son diferentes. Mientras el diseño suele entenderse epistemológicamente como una ciencia social o socio-tecnológica, la física o la biología son entendidas como ciencias naturales o "ciencias duras." Esta compartimentación no es ajena a otras disciplinas, sobre todo en academia y áreas de investigación. El conocimiento humano se ha caracterizado por esta estructuración de las especialidades, y aunque la especialización ha permitido grandes avances en la ciencia, la tecnología y las artes, también ha aislado y limitado las posibilidades de integración del conocimiento con 
orientación teórico-práctica. El resultado de esta falta de integración multidisciplinaria ha derivado en el paradigma del antropocentrismo que experimentamos en el siglo XXI, en particular una visión de diseño que propone un mundo de artefactos y comportamientos completamente desconectados de las maneras en que la naturaleza funciona.

Una nueva generación de diseñadores ha entendido que esta situación pone al diseño como parte de los problemas de este siglo y no como una manera de resolverlos. Estos nuevos actores han encontrado que una perspectiva biocéntrica permite a las disciplinas del diseño evolucionar hacia un futuro menos antropocéntrico y más conectado con las maneras que la naturaleza, las formas de vida y las leyes de la física proponen. Cuando el diseño es "informado" y "guiado" por la naturaleza, los diseñadores pasan de ejercer una práctica a ejercer una praxis, de ser meros ejecutores a ser profesionales reflexivos. En este sentido, una perspectiva biocéntrica permite acercar e integrar el diseño con disciplinas que de otra forma no se conectarían fácilmente. Una de estas disciplinas emergentes es hoy conocida como biomimesis o biomimética (en ingles biomimicry). La biomimesis permite que, por ejemplo, biólogos y diseñadores trabajen en una "misma mesa" como parte de la metodología biomimética.

El trabajo de doctorado del cual este proyecto es parte, se ha nutrido de la idea del biocentrismo para generar nuevas espacios de encuentro entre el diseño y las ciencias que estudian la naturaleza, en particular -desde un interés en la comunicación visual- en estudios y maneras sustentables de producir color. Como parte de este proyecto se han creado herramientas de integración multidisciplinaria que podrían contribuir a desarrollar mejores métodos de investigación y trabajo para la biomimesis, así como también nuevos canales de comunicación entre científicos y diseñadores con interés en implementar la biomimesis de manera tangible. Este artículo describe el proceso que conllevó este proyecto y ofrece reflexiones sobre presente y futuro de esta experiencia.

\section{Biomimesis y biocentrismo}

3.800 millones de años de selección natural y evolución conllevaron a un sistema de sistemas, una red de colaboración y mutualismo densamente entrelazada, a la cual llamamos "vida" (Woolley-Barker, 2013). Todas las formas de vida que conocemos (y las que aún no se han descubierto) son "soluciones de diseño" perfectamente adaptadas para la supervivencia en este planeta, mientras que los restos fósiles que hoy encontramos en sedimentos geológicos, son evidencia de aquellas "soluciones de diseño" que no funcionaron (Benyus, 1997; Wilson, 1984). La "vida" como sistema provee el "programa de diseño" para crear las condiciones que conducen a continuar la vida (Fiorentino, 2018; Benyus, 1997).

Otto Schmidt introdujo el término biomimética en 1954, y lo definió como el estudio sobre la formación, estructura o función de sustancias biológicamente producidas, y de materiales para aplicar en ingeniería y productos (Harkness, 2002). Tres décadas después, en 1984 E.O. Wilson introduce la teoría de bioafiliación (Biophilia, Wilson, 1984) que propone enfatizar nuestra "inherente inclinación humana hacia afiliarnos con los sistemas y procesos naturales, especialmente la vida y las características vivas de los ambientes no-humanos” (Kellert, 
Heerwagen y Maador, 2008, p.3). La teoría de biophilia inspiró el concepto de biomimicry o biomimesis (de bios = vida, y mimesis = imitar), definido como "innovación de diseño inspirado por la naturaleza” (Benyus, 1997). La biomimesis es una disciplina emergente reconocida por su original metodología, con la habilidad de conducir hacia la innovación sustentable (Kennedy y otros, 2016). La biomimesis estudia "el carácter de la naturaleza" y emula conscientemente los principios de adaptación y supervivencia de la naturaleza, imitando no solo las formas, las funciones y las estructuras, pero también los procesos y contextos (Benyus, 1997) entendiéndoles a nivel sistémico. Un entendimiento a nivel sistémico significa una manera de hacer y fabricar en red ("networking") que reemplaza la manera linear y antropocéntrica tradicional. También se vincula a un modelo regenerativo que, como la naturaleza, en vez de solamente consumir materiales vírgenes, también genera la producción de materiales nuevos ("upcycling") en un ciclo virtuoso (Wahl, 2016).

Basado en estas premisas, este proyecto se propuso investigar una de las áreas de diseño más atractivas de la biomimesis: la producción del color en la naturaleza. Mas específicamente, como el color en la naturaleza se manifiesta en forma diferente a la manera que la industria humana lo produce. El "color estructural" es una de estas alternativas.

\section{Colores Sustentables}

El fenómeno del color ha sido largamente estudiado desde múltiples ángulos y disciplinas durante siglos, ofreciendo gran número de estudios, convenciones, estándares y clasificaciones taxonómicas. Sin embargo, solo un puñado de estas convenciones y estándares fueron concebidos con rigor científico; la mayoría enteramente basados en la observación limitada de la percepción visual humana. Aun menos estudios han sido dedicados a otras formas de coloración alternativas que no sean pigmentarias, y en este área el rigor científico es todavía más importante.

Aristóteles estaba convencido que la naturaleza no hace nada sin un propósito. Siguiendo este principio, observar el fenómeno del color en la naturaleza conduce a interesantes preguntas desde la perspectiva del biocentrismo, como por ejemplo:

¿Cuál es el propósito del color presente en las formas de vida?

¿Que estrategias naturales se nutren de la habilidad de ver color, por qué y para qué?

¿Por qué las especies evolucionaron de tener pigmentación a tener otras formas de producir color?

¿Por qué especies tan diversas y disimiles, con diferentes estímulos externos y de ecosistemas tan variados, convergen en similares mecanismos de producir color?

¿Por qué diferentes mecanismos de coloración se observan en especies de aspecto idéntico? ¿Como pueden todos estos argumentos evolucionarios ser explicados en términos comparativos a métodos de diseño?

La lista de preguntas se extiende en muchas direcciones a medida que más investigamos estas cuestiones y el diseño biomimético se involucra. 
El color en la naturaleza ha evolucionado para ser frugalmente generado y usado con eficacia, además de reunir-como la mayoría de las soluciones en la naturaleza-los estándares más altos de sustentabilidad. Después de todo, ¿qué puede ser más sustentable que la naturaleza? (Fiorentino, 2018). Los estándares de diseño sustentable exigen considerar los factores medioambientales, sociales y económicos, para lograr resultados equilibrados con recursos posibles, viables y durables (Fiorentino, 2014) ${ }^{1}$. Los sistemas naturales de producción cumplen con estos estándares, y la manera en que el color se produce también. Los procesos para generar color observados en distintas formas de vida hacen que las más sofisticadas tecnologías creadas por el ser humano parezcan rudimentarias en comparación. El color estructural-en física "interferencia lumínica"-es una de las maneras en que la naturaleza produce color sin basarse en químicos tóxicos, generar polución y usar procesos de producción derrochadores de energía y recursos materiales.

\section{¿Qué es el color estructural?}

El color observado en la naturaleza se obtiene de dos formas: por pigmentación o por estructura. Desde el punto de vista de la física y la química, y la segunda ley de la termodinámica ${ }^{2}$, los pigmentos pueden ser considerados "componentes activos" mientras que el color estructural es una "estrategia pasiva." Los pigmentos (contenidos en minerales, elementos químicos, en materiales naturales o sintéticos) tienen color debido a que ciertos electrones en estado de excitación absorben ciertas longitudes de onda electromagnética de la luz y emiten otras (Hsiung, Siddique, Jiang, Liu, Lu, Shawkey, y Blackledge, 2016). Esta actividad de intercambio de energía está ausente en los mecanismos del color estructural, el cual es producido por superficies incoloras de escala nanométrica, con detalles más pequeños que las ondas electromagnéticas de la luz que producen los diferentes colores. Una ventaja comparativa del color estructural con el color de pigmento es que el primero no se desvanece con el tiempo o la descomposición del material biológico, como si sucede con los pigmentos.

El color estructural está en el cruce entre la física y la biología (biofotónica) ${ }^{3}$ y ha sido observado por más de tres siglos desde su descubrimiento y las primeras descripciones científicas hechas por Hooke y Newton en 1704 (Kinoshita, 2008). Sin embargo, solo recientemente y con el avance de la computación, y la micro y nanotecnologías, ${ }^{4}$ el color estructural parece alcanzable como método para nuevas tecnologías de coloración.

En física el fenómeno se conoce como "interferencia lumínica” y en biomimesis también como "colores sin pigmento" (Benyus, 1997). En muchas especies observadas el color estructural es conseguido con "información agregada" a la superficie de los materiales a escala manométrica. La mayoría de los mecanismos y estructuras observadas se pueden definir como "variaciones de la luz dispersándose al encontrarse con objetos que difieren en su índice refractivo" (Prum y Torres, 2003), es decir donde la luz incidente rebota desde la nanoestructura de la superficie en forma de diferentes colores (fig.1). 

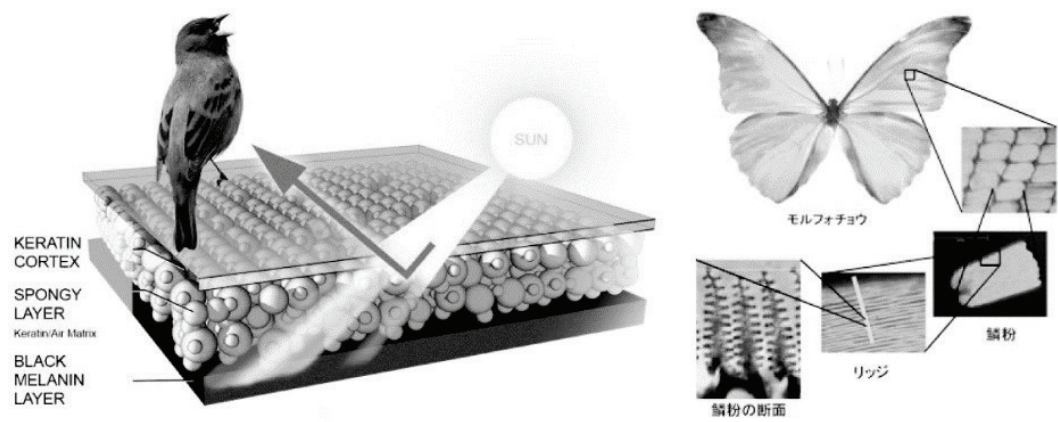

Figure 1. Diagramas que explican la difracción de luz azul en aves y lepidóptera (mariposas), debido a las nanoestructuras observadas en los mecanismos de color estrucutral.

Es importante hacer una distinción entre color estructural producido por "dispersión coherente" versus "dispersión incoherente" de la luz. En la dispersión incoherente el color es una función de las propiedades de dispersores individuales (por ejemplo, moléculas de agua o moléculas de oxígeno, que haces que el hielo, la nieve o el cielo luzcan azules), mientras que en la dispersión coherente el color es determinado por la distribución espacial de las interfaces dispersoras de luz (Parker, 1998), como el color de las plumas en algunas aves o el de las escamas en peces. El interés de la biomimesis en color estructural se enfoca en esta última, sin embargo nuevos descubrimientos y variaciones en los mecanismos de dispersión de luz observados podrían sugerir más formas de biomimetismo del color (Seago y otros, 2009).

El color estructural dado por dispersión coherente es abundante entre las formas de vida. Observado en animales (Parker, 1998; Prum y otros, 2003 y 2004; Saranathan, Seago, Sandy, Narayanan, Mochrie, Dufresne, Chinedum, Osuji, y Prum, 2015; Seago, Brady, Vigneron, y Schultz, 2009; Vukusic, 2004), plantas (Jacobs, Lopez-Garcia, Phrathep, Lawson, Oulton, y Whitney, 2016; Vignolini, Rudall, Rowland, Reed, Moyroud, Faden, Steiner, 2012), y hongos y bacterias (Starkey \& Vukusic, 2013; Hsiung y otros, 2017). Casos abundantes de color estructural en aves, peces y artrópodos han sido estudiados por décadas, pero nuevos aspectos y variaciones de lo que la ciencia conoce esperan aun por ser descubiertos (Vukusic, 2004; Saranathan y otros, 2015).

\section{Aplicaciones e implicaciones del color estructural}

La premisa tan utilizada en diseño "la forma sigue a la función" es también observada en la naturaleza; el propósito de estas sofisticadas estructuras manométricas para producir color, desarrolladas después de un largo proceso de evolución y optimización, está en la 
esencia de la "metodología" de las formas de vida, que es sobrevivir. Basado en su distribución filogenética, el color estructural de las aves por ejemplo, ha evolucionado convergentemente más de 50 veces dentro de una misma especie (Prum y Torres, 2003), hasta las aves que podemos observar hoy con colores que tal vez no son los mismos que los de sus ancestros. Aprender de estas adaptaciones, cuando y para que sucedieron, pueden dar pistas para desarrollar nuevas ideas de innovación sustentable en industrias del color, óptica, computación y energía - pinturas, tintas, textiles, pantallas electrónicas, film fotovoltaicos, lentes, etc.- (Vukusic, 2004; Saito et al, 2011; Xiao et al, 2015; Saranathan et al, 2015).

La manera de producir color en la industria del diseño heredada del siglo XX ha sido enfocada en dos procesos diferentes, y fundamentalmente dañinos en términos ecológicos:

- La mezcla sustractiva por pigmento, basada en extracción de minerales y uso de químicos-usualmente tóxicos, y procesos de gran consumo de energía para la transferencia y fijación del color. Esta manera de producir color es altamente contaminante, utiliza una enorme cantidad de agua, genera desperdicios y es ineficiente. Mientras que la industria se ha enfocado en la exactitud de la reproducción de color y su estandarización (por ejemplo los estándares Pantone y CMYK en sistemas de impresión), los resultados de color en materiales permanecen a veces impredecibles o imposibles de reproducirse con 100\% de exactitud. Además, los colores pigmentarios son entrópicamente inestables (segunda ley de la termodinámica antes mencionada) y cambian y se desvanecen con el tiempo en condiciones normales de iluminación.

- La mezcla aditiva de luz o pixeles, observada en pantallas electrónicas, está basada en materiales fosforescentes y minerales exóticos-como el tungsteno, oro, mercurio, etc. Estos materiales son también muy tóxicos y contaminantes, especialmente si se tiene en cuenta la cantidad masiva de electrónicos producidos a escala mundial, y la basura electrónica generada por la pronta obsolescencia de artículos como smartphones, TV y computadoras.

En contraste a estos dos procesos insustentables, las 8.700 .000 especies que se estiman en el planeta, cuando logran su coloración lo hacen de manera sustentable, es decir con 100\% de los materiales biodegradables y reciclables, con bajo consumo de energía (usualmente energía solar), hecha con abundantes y simples materiales, localmente desarrollados, y creados con la combinación de unos pocos elementos de la tabla periódica, y en condiciones ambientales normales. Esta manera "primitiva" de crear color es una de las lecciones más grandes de sofisticación e "inteligencia” que podemos aprender de la naturaleza, como lo sugiere la biomimesis.

La implementación de color estructural jugara un rol crucial en el prospecto de nuevas tecnologías sustentables (Caro, Stoddard, and Stuart-Fox, 2017; Hsiung, y otros, 2017; Kennedy, y otros, 2015; Kinoshita, 2008; Saito, y otros, 2011; Xiao, y otros 2015), por ejemplo en la industria electrónica, la impresión, comunicaciones, computación fotónica, entre otras. Sin embargo, los mecanismos de color estructural son complejos de imitar artificialmente con los actuales métodos de fabricación. Estos mecanismos pueden ser abordados desde la nanotecnología (Zhang y otros, 2015), la fabricación aditiva $\neg-3 \mathrm{D}$ printing (Boyle y otros, 2017), y otras formas digitales de fabricación (Colusso y otros, 
2017); pero estas técnicas no están completamente desarrolladas aun y siguen en permanente evolución. Es así que el color estructural ha sido aplicado solo en un puñado de productos y prototipos, en los últimos 10 años (fig.2). Hay todavía mucho que aprender de la naturaleza y varias limitaciones técnicas que superar antes de ver casos más exitosos beneficiados por color estructural.

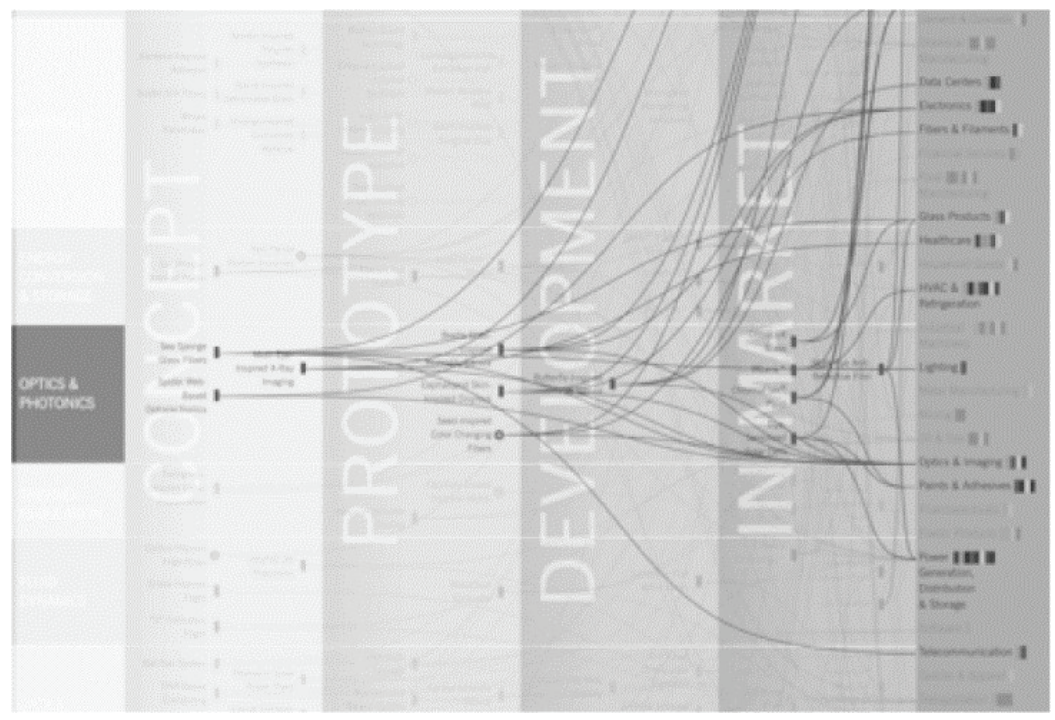

Figura 2. Esta tabla muestra el número de casos de biomimetica, óptica y fotónica (2015), que pasaron la etapa de prototipo hacia la etapa de producción. Sólo cuatro de estos casos llegaron a ser productos piloto lanzados al mercado en más de 10 años (Tapping into Nature, Terrapin Bright Green LLC Report, 2015 by terrapin.org)

Una de las mayores limitaciones de reproducir color estructural es la capacidad de obtener colores estables, independientes del ángulo de incidencia de la luz o el punto del observador. Cuando este efecto sucede, percibimos dichos colores con iridiscencia o efectos metálicos. Esta limitación hace que el color estructural no sea apto para reemplazar algunas aplicaciones. Sin embargo descubrimientos científicos recientes, observando color estructural en especies antes no exploradas, abren nuevas posibilidades para desarrollar tecnologías que superan actuales limitaciones (Hsiung, 2017; Saito y otros, 2011; Saranathan y otros, 2015; Starkey \& Vukusic, 2013; Xiao y otros, 2015). La perspectiva para investigar en innovar no podría ser más prometedora. 


\section{Un buscador de prospecto mejorado, y prototipeado académico como mé- todo de investigación}

Investigar e innovar sobre color estructural requiere de herramientas que permitan acceder al conocimiento científico, a información existente y actualizada de fuentes con rigor científico. También requiere que esta información llegue en forma entendible para investigadores e innovadores fuera del dominio científico, como es el caso de los diseñadores e innovadores de la biomimesis. En este sentido, la experiencia personal y contacto con la comunidad científica experta en el área de la biofotonica, mediante la participación en conferencias especializadas en el tema, permitieron detectar una gran necesidad de conectar los actores del conocimiento con los actores de la innovación, problema todavía no abordado por ninguna herramienta de investigación concreta.

Es así como este proyecto de doctorado propone como metodología la creación de dichas herramientas, para proporcionar un espacio de intercambio entre científicos y diseñadores sobre el tema color estructural. El proyecto incluye un estudio comprehensivo para establecer la efectividad de las herramientas de investigación creadas. El método utilizado para conducir dicho estudio combina dos conceptos: el prototipeado académico (Ruecker, Adelaar, Brown, y Dobson, 2014) y el buscador de prospecto mejorado Ruecker, 2003; Ruecker, Radzikowska, y Sinclair, 2011); el primero asociado con métodos etnográficos, el segundo con el análisis hermenéutico (Rockwell y Sinclair, 2016). El prototipeado académico es una manera de producir nuevo conocimiento sobre ideas especificas a través del proceso de diseño y desarrollo de un prototipo; el objetivo no es solo la producción de dicho prototipo sino también experimentar el prototipeado como una fase del proceso crítico (Ruecker y otros, 2014). Un buscador de prospecto mejorado es una interface experimental la cual representa visualmente cada ítem de una colección dada, combinada con herramientas de manipulación (Ruecker, 2003). Estos dos métodos son complementados con una variedad de técnicas en visualización de datos, diseño de la información y organización taxonómica (Tufte, 1983), usualmente combinados en el campo de las humanidades digitales (digital humanities) y estudios de la información para el análisis de datos. La herramienta digital creada para el estudio propuesto es una interface digital utilizada como un "probe" o "sonda de prueba" (Gaver, Boucher, Pennington, y Walker, 2004; Mattelmäki, 2005), lleva el nombre StrC (siglas para "Structural Colour"), y puede definirse como un buscador taxonómico de prospecto mejorado. Las"probes" al igual que el prototipeado académico, proponen descubrir partes invisibles de un problema (Gaver y otros, 2004). El uso de una interface digital como probe sirve como herramienta de intercambio y comunicación entre el diseñador y el usuario final de dicha herramienta (Mattelmäki, 2005). Asi, StrC será "enviada" como un "objeto digital" a los participantes del estudio, quienes en retorno usaran, interactuaran, crearan, y enviaran comentarios al investigador/administrador.

\section{StrC study: conectar el conocimiento científico con el diseño innovador}

StrC se compone de una base de datos autoadministrada, la interface digital (similar a un website) y varios componentes de visualización de datos (denominados "widgets") 
(fig.3). La estrategia del estudio propone el rigor de la información taxonómica (de Hoog, 1981) combinada con la flexibilidad, interactividad y efectividad visual de un buscador de prospecto mejorado. La interface de StrC provee acceso a información científica confiable sobre color estructural, organizada por niveles de relevancia, y permite al usuario agrupar, discriminar y customizar la información recolectada en forma flexible e intuitiva. El objetivo del estudio es testear la herramienta con diseñadores y científicos, y evaluar la efectividad en conectar el conocimiento científico con la posible implementación de diseño. La base de datos de StrC fue inicialmente poblada con un set de datos pequeño manualmente administrado, pero está preparada para acceder en el futuro cercano a grandes repositorios de datos y fuentes de información científica (por ejemplo EOL.org, ITIS. gov, iNaturalist.org, WebOfScience.org, entre otros).

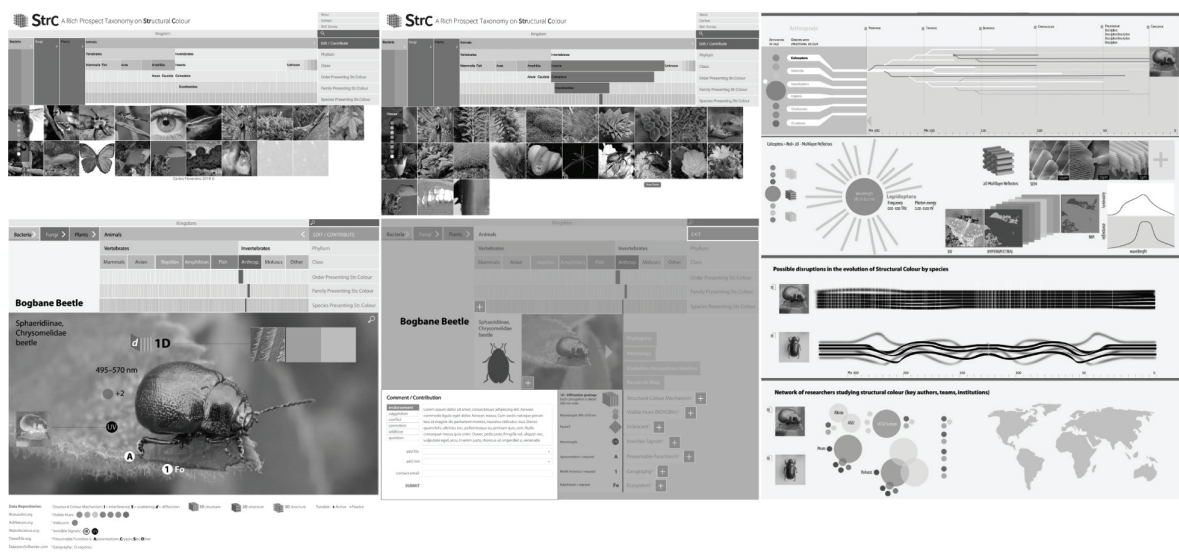

Figura 3. Screenshots de la interface StrC, que muestran el acceso a la colección taxonómica por color (arriba de izquierda a derecha) y funciones principales del perfil de especies (abajo de izquierda a derecha); y los “widgets" de manipulación y visualización de datos (a la derecha).

Los principales participantes del estudio serán diseñadores y científicos. Los diseñadores podrán identificar oportunidades para la innovación, basadas en el estudio de la interacción de la especies a través del uso de color estructural (por ejemplo en emisión de señales, estímulos, mutualismos y reciprocidad), mientras que los científicos podrán identificar patrones de coincidencia, convergencias y hacer observaciones filogenéticas y evolucionarias. Los participantes tendrán acceso a la interface y serán invitados a experimentar con ella, guiados por una lista de preguntas y guías para testear el potencial de la herramienta más allá de la etapa de prototipo. StrC colectará datos (anónimos) generados por la actividad de los participantes al navegar la interface; estos datos proveerán evidencia sobre patrones de uso, comonalidades, diferencias y otros importantes rasgos para identificar oportunidades para futuro desarrollo. 
StrC también brindará a los científicos la oportunidad de contribuir con comentarios y sugerencias para mejorar la herramienta, así como también aportar nuevos casos de color estructural y acceso a nuevos avances científicos sobre el tema. Para ello, la interface tiene una función que recolecta el feedback para posterior revisión.

Al finalizar la exploración de la interface StrC, los participantes serán invitados a completar una encuesta online autoadministrada con una serie de preguntas relativas a la efectividad de los componentes y funciones de StrC, dejando abierta la posibilidad de agregar comentarios adicionales que ayuden a mejorar y desarrollar la herramienta en el futuro. Las respuestas a esta encuesta serán analizadas de manera cuantitativa y cualitativa, y apuntaran a encontrar evidencia que soporte o desafíe la hipótesis general del estudio.

\section{Hipótesis}

La hipótesis general que este estudio corroboraría o modificaría se basa en la experiencia y contacto del investigador con la comunidad científica experta en el área de la biofotónica, y es que la necesidad de conectar los actores del conocimiento científico con los actores de la innovación del diseño biomimético es un problema no abordado convenientemente por ninguna herramienta de investigación existente. Las fisuras en la comunicación, o directamente la falta de comunicación entre las disciplinas científicas y las de diseño sobre el tema de color estructural crean limitaciones para acceder y entender el material científico desde la perspectiva del diseño. Estas limitaciones pueden ocurrir debido a las diferentes vistas epistemológicas entre disciplinas. La dificultad para acceder y entender la información científica debe ser una de las principales limitaciones que impide el progreso de ideas innovadoras.

\section{Justificación del estudio}

La facilidad de acceso a la información de rigor científico que diferentes actores de diferentes campos disciplinarios puedan comprender, hace pensar en posibles resultados de este estudio de investigación aproximándolo desde un ángulo de facilitación y mediación. Esta aproximación invita a la exploración y la experimentación, y permite descubrir qué más puede ser aprendido o está esperando por ser descubierto de la naturaleza, y que podría brindar nueva evidencia para crear, manipular y usar colores sin pigmento. Esto también podría revelar nuevas maneras de acceder y compartir conocimiento con una audiencia más amplia interesada en el tema. Como materia primordial, este proyecto de investigación puede también contribuir a consolidar una teoría de diseño biocentrista que permita a las disciplinas del diseño evolucionar de las practicas antropocéntricas insustentables. 


\section{Objetivos del estudio}

Este estudio de investigación proveerá pistas sobre cómo mejorar la conexión entre la información científica existente de color estructural y la innovación y práctica de diseño en biomimesis del color. El estudio puede revelar patrones de conducta, tendencias, comonalidades, oportunidades y características ocultas en los dos campos (científico y de diseño), y podría confirmar o desafiar asunciones e hipótesis previas. El estudio invitará a participantes científicos a explorar y contribuir al Sistema StrC. Como la herramienta está inspirada en el prototipeado académico (que implica un proceso abierto al mejoramiento permanente), algunas contribuciones hechas durante el estudio podrían ser incorporadas al diseño de la herramienta en una etapa posterior; con la posibilidad de pedir a los científicos más opiniones sobre estas actualizaciones. Diseñadores e investigadores interesados en el diseño biomimético también serán invitados a explorar StrC. Crear sinergias entre científicos, investigadores y diseñadores, y detector intereses comunes será un plus para este estudio.

Los procedimientos, recolección de datos y análisis del proyecto se resumen en las siguientes fases:

- Fase 1 (preliminar): Diseño, programación e implementación del prototipo de contexto digital StrC

- Fase 2 (preliminar): Testeo técnico de StrC (sin participantes)

- Fase 3: Estudio: participantes exploran StrC online y completan el cuestionario online (opcionalmente también pueden programarse entrevistas personales)

- Fase 4: Análisis de datos asistido por herramientas hermenéuticas digitales

- Fase 5: Reporte y diseminación del estudio en forma de disertación de doctorado y publicación de artículos en publicaciones especializadas del ámbito científico y de diseño biomimético.

\section{Futuros pasos}

La comprensión de los mecanismos de color en la naturaleza, con ayuda de nuevas tecnologías, permitirá a científicos y diseñadores acceder a información que medio siglo atrás era todavía un terreno inalcanzable de explorar. Aún lejos de ser totalmente entendido y ser mayormente inexplorado, el prospecto para producir color de manera sustentable está cambiando la visión de científicos y diseñadores hasta niveles filosóficos. El uso de StrC contribuirá al progreso del conocimiento en este sentido.

Futuras fases del Proyecto estarán orientados a consolidar la herramienta e incluirán estudios de usuario (UX), extenderán el acceso a otras bases de datos y repositorios, y el análisis de nuevos datos. Las derivaciones de este proyecto de investigación, más allá de la disertación de doctorado, implican publicar una serie de artículos sobre los resultados del análisis de datos y la experiencia sobre el uso de StrC como herramienta de investigación; así como también se diseminará el caso en conferencias del ámbito de la biomimesis, la in- 
novación de diseño, la biofotónica, y la innovación en tecnología y materiales. Otras fases del proyecto se enfocaran en hacer que el sistema StrC sea accesible libremente o mediante suscripción libre a investigadores, académicos, diseñadores y científicos.

\section{Notas}

1. "What is More Sustainable Than Nature? Anthropocentric and Biocentered Design." Presentación en la 14th. International Conference on Environmental, Cultural, Economic \& Social Sustainability, el 17-de enero del 2018, The Cairns Institute, James Cook University, Australia.

2. La segunda ley de la termodinámica explica la transferencia de energia y la perdida de calor entre la materia de forma natural (entrópica) que tiende al equilibrio de fuerzas, y establece la irreversibilidad de este proceso.

3. Ibid

4. Entre las tecnologías actuales para detectar y medir color estructural existen Refractive Index Matching, Transmission Electron Microscopy (TEM) y Scanning Electron Microscopy (SEM).

5. La investigación actual sobre una nueva generación de transistores permitirá usar fotones en lugar de, o combinados con, electrones para producir dispositivos fotónicos, 100 veces más rápido que los electrónicos, lo que puede revolucionar la ciencia de la computación y abrir nuevas oportunidades para explorar la computación cuántica. (http://www. sciencealert.com/scientists-have-figured-out-how-to-switch-between-electrons-andphotons-in-a-single-transistor).

\section{Lista de Referencias Bibliográficas}

Benyus, J.M. (1997) Biomimicry: innovation inspired by nature. New York: Perennial.

Boyle, B. M., French, T. A., Pearson, R. M., McCarthy, B. G., \& Miyake, G. M. (2017). Structural Color for Additive Manufacturing: 3D-Printed Photonic Crystals from Block Copolymers. ACS Nano, 11(3).

Brown, S., Adelaar, N., Dobson, T., Knechtel, R., MacDonald, A., Nelson, B., and the Inke Group. (2014). Probing Digital Scholarly Curation through the Dynamic Table of Contexts. Conference paper for the Digital Humanities 2014 Annual Conference, McGill University.

Caro, T., Stoddard, M. C., \& Stuart-Fox, D. (2017). Animal coloration research: why it matters. Philosophical Transactions of the Royal Society B: Biological Sciences, 372(1724). https://doi.org/10.1098/rstb.2016.0333

Colusso, E., Perotto, G., Wang, Y., Sturaro, M., Omenetto, F., \& Martucci, A. (2017). Bioinspired stimuli-responsive multilayer film made of silk-titanate nanocomposites. J. Mater. Chem. C, 5(16). https://doi.org/10.1039/C7TC00149E

de Hoog, G. S. (1981). “Methodology of Taxonomy." Taxon 30 (4), 779-783.

Fiorentino, C. (2018). Transición del Antropocentrismo al Biocentrismo: El Presente Distopico y el Futuro del Diseño. IX Congreso Latinoamericano de Enseñanza del Diseño, 31 
July, 2018 Congreso de Enseñanza del Diseño Universidad de Palermo, Buenos Aires, Argentina.

Fiorentino, C., Montana-Hoyos, C. (2014). "The Emerging Discipline of Biomimicry as a Paradigm Shift towards Design for Resilience." The International Journal of Designed Objects. Champaign, Illinois, USA: Com- mon Ground Publishing LLC 8 (1) 2-15.

Gaver, W. W., Boucher, A., Pennington, S., \& Walker, B. (2004). "Cultural probes and the value of uncertainty." Interactions, 11(5), 53-56.

Harkness, J. (2002). “A Lifetime of Connections: Otto Herbert Schmitt, 1913-1998," Physics in Perspective 4: 456-90.

Hsiung, B., Justyn, N., Blackledge, T., Shawkey, M. (2017). "Spiders have rich pigmentary and structural colour palettes." The Journal of experimental biology 2017 vol: 220 (Pt 11) 1975-1983.

Hsiung, B.K., Siddique, R., Jiang, L., Liu, Y., Lu, Y., Shawkey, M., and Blackledge, T. (2016). "Structural Colors: Tarantula-Inspired Noniridescent Photonics with Long-Range Order" Advanced Optical Materials 5 (2).

Jacobs, M., Lopez-Garcia, M., Phrathep, O. P., Lawson, T., Oulton, R., \& Whitney, H. M. (2016). Photonic multi-layer structure of Begonia chloroplasts enhances photosynthetic efficiency. Nature Plants, 2. https://doi.org/10.1038/nplants.2016.162

Kellert, S.R., Heerwagen, J. and Mador, M. (2008). Biophilic Design: The Theory, Science and Practice of Bringing Buildings to Life. Hoboken, NJ: Wiley.

Kennedy, E. B., \& Marting, T. A. (2016). "Biomimicry: Streamlining the Front End of Innovation for Environmentally Sustainable Products." Research-Technology Management, 59(4), 40-48.

Kennedy, E., Fecheyr-Lippens, D., Hsiung, B. K., Niewiarowski, P. H., \& Kolodziej, M. (2015). "Biomimicry: A path to sustainable innovation." Design Issues, 31(3), 66-73.

Kinoshita, S. (2008). Structural colors in the realm of nature. New Jersey: World Scientific. Mattelmäki, T. (2005). "Applying Probes: from Inspirational Notes to Collaborative Insights." CoDesign 1 (2): 83-102.

Parker, A R. (1998). “The Diversity and Implications of Animal Structural Colours.” The Journal of Experimental Biology 201(16), 2343-47.

Prum, R. O. and R.H. Torres (2003). "Structural Colouration of Avian Skin: Convergent Evolution of Coherently Scattering Dermal Collagen Arrays." Journal of Experimental Biology 206 (14), 2409-29.

Prum, R. O. and R.H. Torres (2004). "Structural Colouration of Mammalian Skin: Convergent Evolution of Coherently Scattering Dermal Collagen Arrays.” Journal of Experimental Biology 207 (12), 2157-72.

Rockwell, G., \& Sinclair, S. (2016). Hermeneutica: Computer-Assisted Interpretation in the Humanities. MIT Press.

Ruecker, S. (2003). Affordances of prospect for academic users of interpretively tagged text collections. University of Alberta (Canada).

Ruecker, S., Brown, S., Radzikowska, M., Sinclair, S., Nelson, T. M., Clements, P., Antoniuk, J. (2009). The Table of Contents: A Dynamic Browsing Tool for Digitally Encoded Texts. In L. Dolezalova (Ed.), The Charm of a List: From the Sumerians to Computerized Data Processing. Cambridge: Cambridge Scholars Publishing, 177-187. 
Ruecker, S, Adelaar, N., Brown, S. and Dobson, T. (2014). "Academic Prototyping as a Method of Knowledge Production: The Case of the Dynamic Table of Contexts." Scholarly and Research Communication 5 (2).

Ruecker, S., Radzikowska, M., and Sinclair S. (2011). Visual Interface Design for Digital Cultural Heritage: A Guide to Rich-Prospect Browsing. Surrey, UK: Ashgate Publishing, Ltd.

Saito, A., Yoshioka S., Kinoshita S., Berthier S., Hooke R., Newton I., Michelson A., Rayleigh J. W. S. (2011). "Material Design and Structural Color Inspired by Biomimetic Approach." Science and Technology of Advanced Materials 12 (6).

Saranathan, V., Seago, A., Sandy, A., Narayanan, S., Mochrie, S., Dufresne, E.,Chinedum, H.C. Osuji, O. and Prum, R. (2015). "Structural Diversity of Arthropod Biophotonic Nanostructures Spans Amphiphilic Phase-Space.” Nano Letters 15 (6). American Chemical Society: 3735-42.

Seago, A. E., Brady, P., Vigneron, J.-P., \& Schultz, T. D. (2009). “Gold bugs and beyond: a review of iridescence and structural colour mechanisms in beetles (Coleoptera)." Journal of the Royal Society, Interface / the Royal Society, 6 Suppl 2(Suppl_2), S165-84.

Starkey, T., \& Vukusic, P. (2013). "Light manipulation principles in biological photonic systems." Nanophotonics, 2(4), 289-307.

Tufte, E. R. (1983). The visual display of quantitative data. Cheshire, CT: Graphics Press.

Vignolini, S., Rudall, P. J., Rowland, A. V, Reed, A., Moyroud, E., Faden, R. B., ... Steiner, U. (2012). "Pointillist structural color in Pollia fruit." Proceedings of the National Academy of Sciences of the United States of America, 109(39), 15712-5.

Vukusic, P. (2004). “Natural Photonics." Physics World 17 (2). Australian Institute of Physics. Wahl, D. C. (2016). Designing Regenerative Cultures. Axminster, UK: Triarchy Press.

Wilson, E. O. (Ed.). (1984). Biophilia: The human bond with other species. Cambridge, MA.: Harvard University Press.

Woolley-Barker, T. (2013) "How Would Nature Create A 'Generous City'?", accessed July 9th, 2013 http://www.triplepundit.com/2013/07/creating-conditions-conducive-lifefirst-biomimicry-38-global-conference/

Xiao, M., Li, Y., Allen, M., Deheyn, D., Yue, X., Zhao, J., Gianneschi, N., Shawkey, M., and Dhinojwala, A. (2015). "Bio-Inspired Structural Colors Produced via Self-Assembly of Synthetic Melanin Nanoparticles." ACS Nano 9 (5). American Chemical Society, 5454-60.

Zhang, S., \& Chen, Y. (2015). Nanofabrication and coloration study of artificial Morpho butterfly wings with aligned lamellae layers. Scientific Reports, 5. https://doi.org/10.1038/ srep 16637

\begin{abstract}
This article is based on the author's doctoral project, and it has roots to biocentered thinking. The project is intended to generate new spaces to meet design with natural sciences, particularly on sustainable ways to produce colour. As part of it, research tools for multidiciplinary integration were created, that may contribute to the development of better research methods for biomimetic design practice. This article describes the process that this project involved, and offers reflections on present and future steps of this experience.
\end{abstract}


Keywords: Structural colour - biocentered design - anthropocentrism - biomimicry, sustainability - multidisciplinarity - academic prototyping - rich prospect browsing.

Resumo: Este artigo é baseado no projeto de doutorado do autor e tem raízes no pensamento biocêntrico. O projeto pretende gerar novos espaços para atender o design com as ciências naturais, particularmente em formas sustentáveis de produzir cores. Como parte disso, ferramentas de pesquisa foram criadas para integração multidisciplinar, o que pode contribuir para o desenvolvimento de melhores métodos de pesquisa para a prática do design biomimético. Este artigo descreve o processo que este projeto envolveu e oferece reflexões sobre os passos presentes e futuros dessa experiência.

Palavras-chave: Cor estrutural, design biocentrado, antropocentrismo, biomimética, sustentabilidade, multidisciplinaridade, prototipagem acadêmica, rich prospect browsing.

[Las traducciones de los abstracts fueron supervisadas por el autor de cada artículo] 